Received: 2020-05-06 Accepted: 2020-06-23 Published: 2020-11-30

\section{Improvement in Neurogenic Pain WITH EpIDURAL InJeCtIONS Following INTERSPINOUS SPACER IMPLANTATION IN Patients with Lumbar Stenosis: A Case Series}

\author{
Paul So, MD' \\ Steve M. Aydin, DO2,3
}

Background: Lumbar spinal stenosis with neurogenic claudication can be a debilitating condition, affecting quality of life. Interspinous spacer implantation is a minimally invasive procedure for treatment of lumbar spinal stenosis with neurogenic claudication and associated symptoms by minimizing spinal extension and therefore neural compression.

Case

Presentation: This case series presents 4 cases of patients with multilevel stenosis, most radiographically severe in the lumbar region, all who received interspinous spacers at L3-4 and L4-5 after minimal improvement in symptoms with conservative management including epidural injections. In all 4 cases, patients reported improved standing and gait but limited improvement in pain and overall function after interspinous spacer implantation. Each patient underwent repeat epidural injections at or below the level of the interspinous spacer with significant improvement in pain for up to 6 months.

Conclusion: $\quad$ Our conclusion is that either postspacer epidural injections helped reduce inflammation associated with the implantation procedure, or the spacer maintained an open space to allow the injectate to permeate areas with the most stenosis and help reduce inflammation and therefore pain.

Key words: Epidural injection, interspinous process decompression, interspinous spacer, interspinous spacer implant, low back pain, lumbar spinal stenosis, neurogenic claudication, spinal stenosis

\section{BACKGROUND}

Lumbar spinal stenosis (LSS) is a major cause of pain and disability, as well as the primary reason for spinal surgery in patients 65 years of age and older (1). Neurogenic claudication and low back pain are the most common presenting symptoms in patients with LSS (2).
Although neurologic examination may remain normal, some patients exhibit motor weakness and sensory loss in one or more nerve root distributions. Additionally, functional deficits such as gait abnormalities may also be present. In the majority of patients, magnetic resonance imaging (MRI) typically reveals degenerative

From: ${ }^{1}$ Department of Physical Medicine and Rehabilitation, Rutgers New Jersey Medical School,Newark, NJ; ${ }^{2}$ Department of Physical Medicine and Rehabilitation, Zucker School of Medicine at Hofstra/Northwell, Hempstead, NY; ${ }^{3}$ Kayal Orthopaedics, P.C., Glen Rock, NJ 
compromise of the central canal, lateral recess, and/or neural foramina leading to stenosis (3). This narrowing has been shown to compress the nerve roots and to be exacerbated with lumbar extension (4). Thus, symptoms may typically be elicited on physical examination with lumbar extension (5).

The goals of management in patients with LSS are pain relief and functional improvement. Treatment typically begins conservatively with medical management, including oral analgesics and anti-inflammatory agents, in addition to physical therapy. In patients with persistent symptoms, epidural steroid injections may be considered prior to surgical referral (6). Decompressive laminectomy with or without fusion is an elective procedure and the most frequently utilized approach for LSS. It is typically reserved for patients who fail conservative management. However, patients may be poor surgical candidates or refuse invasive decompression surgery altogether.

Interspinous spacer implantation is a minimally invasive, indirect decompression treatment option available for patient with LSS and symptomatic neurogenic claudication without evidence of spondylolisthesis $(7,8)$. Under fluoroscopic guidance, the device is implanted between the spinous processes at one or 2 levels; implantation can be performed under local and intravenous sedation anesthesia and as a same-day procedure (9). Additionally, it has been shown to have faster procedural time with less blood loss compared to surgery (10). Studies have shown symptomatic relief and improvement in disability after implantation, maintained at 2-, 4-, and 5-year follow-ups (11-14). Furthermore, decompression with an interspinous spacer is associated with a reduction in opioid use (15).

In some cases, patients may have less than expected improvement after the procedure for a variety of reasons. In this limited case series, we discuss 4 cases of patients with interspinous spacer implantation with minimal pain relief and their subsequent responses to postimplant epidural injections. We also discuss the possible mechanisms of these responses and potential options for future management.

\section{CASE SERIES}

This is a limited case series of 4 patients with a history of lumbar spinal stenosis with neurogenic claudication. All 4 patients initially presented with complaints of low back pain and subjective symptoms of neurogenic claudication including difficulty with standing and ambulation. On examination, patients reported exacerbated low back and radicular pain with lumbar extension and straight leg test. They were also noted to have wide-based gait and difficulty with sit-to-stand. All 4 patients subsequently underwent MRI of the spine and met the criteria for the diagnosis of lumbar spinal stenosis with the most severe stenosis observed at the L3-4 and L4-5 levels (Fig. 1).

The 4 patients underwent 6 months or more of conservative management, which included combinations of oral nonsteroidal anti-inflammatory drugs and neuropathic medications. Additionally, they were referred for physical therapy, which was geared towards general strengthening exercises and conditioning. This was followed by a combination of interlaminar, caudal, and transforaminal epidural injections based on symptoms at examination and in concordance with the MRI findings. There was up to $50 \%$ improvement in neurogenic pain for up to 2 months; however, this decreased to as low as $20 \%$ despite repeat epidural injections.

All patients were then given the option of interspinous spacer placement at the L3-4 and L4-5 levels, which were done under live fluoroscopic guidance. All 4 patients tolerated the procedure well with no complications noted after implantation. Post implant, all patients reported significant functional improvement in standing and gait. In one case, a 100\% improvement in neurogenic claudication was seen for 10 days; however, this declined to $30 \%$ at 3 months' follow-up. In the other cases, patients were noted to have improvement in pain up to $50 \%$ for up to 3 months.

All 4 patients underwent epidural injections at or below the level of the interspinous implant. There was $100 \%$ improvement in neurogenic pain for up to 6 months in one case with interlaminar epidural injection at L5-S1. In a second case, an $80 \%$ improvement was seen for 3 months following bilateral transforaminal epidural injection at L4-5. In the remaining 2 cases, no change was seen with interlaminar epidural injections at L5-S1; however, up to $60 \%$ improvement was seen following bilateral transforaminal epidural injections at L4-5 for up to 6 months. Functional improvement was maintained throughout this period.

\section{DISCUSSION}

Interspinous spacer implants are typically placed at the stenotic vertebral levels. The device acts by maintaining intervertebral height and minimizing lumbar extension at these levels. This, in turn, prevents further 
Fig. 1. MRI sagittal views of normal (left) vs stenotic lumbar spine (right; red arrow).
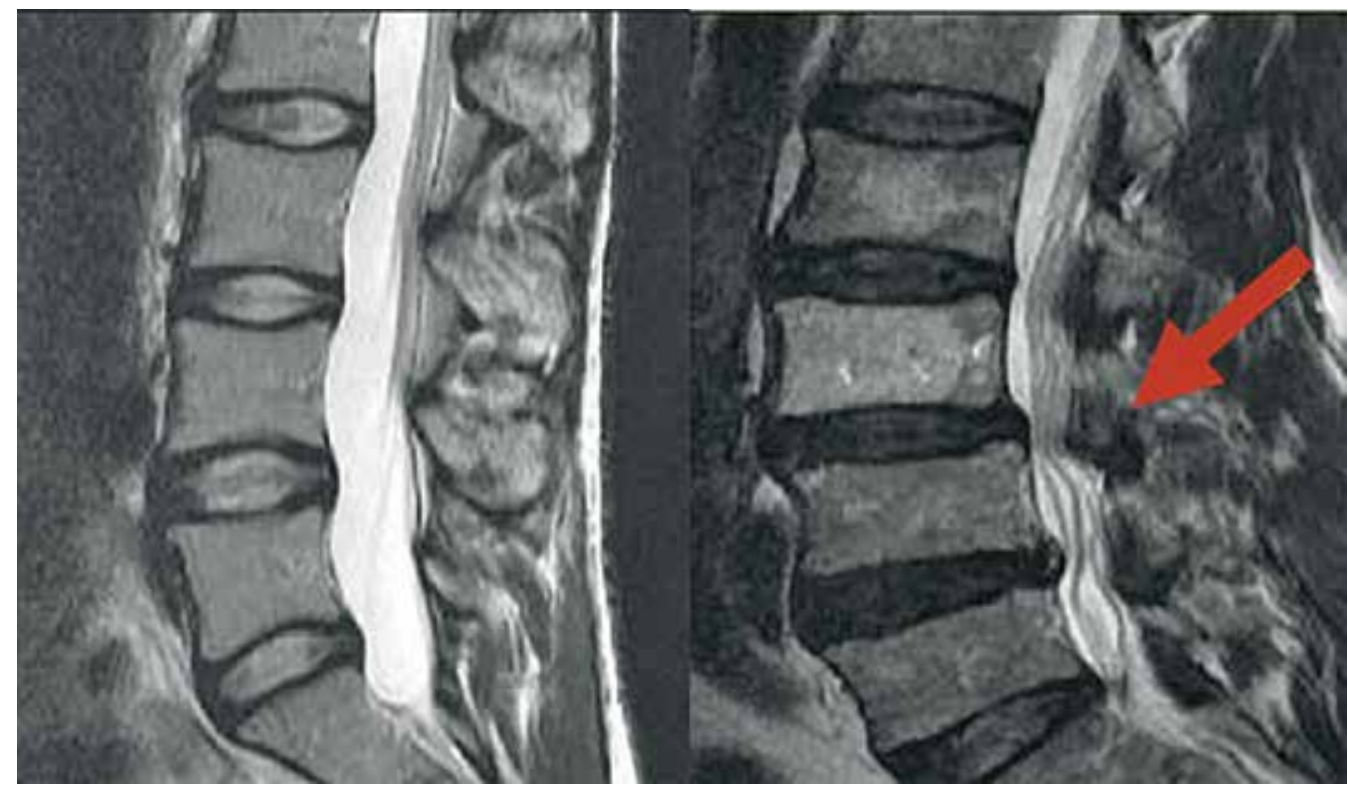

narrowing of the stenotic regions in the central canal and neural foramina with reduction in subsequent neural compression.

This case series has demonstrated improvement in symptoms of neurogenic claudication with epidural steroid injections after implantation of interspinous spacer devices in patients with persistent symptoms. This is likely explained by 2 potential mechanisms. First, the implant maintained an open space to allow the injectate to permeate areas with the most stenosis (Figs. 2 and 3). Lumbar spinal stenosis can result from the narrowing of various structures including the central canal, lateral recess, and neural foramen. Narrowing of the lateral recess and neural foramen can give rise to symptoms of neurogenic claudication as mechanical compression and nerve root ischemia occurs. Central canal narrowing can also lead to increased intrathecal pressure resulting in indirect compression of the nerve roots. Extension of the lumbar spine can further narrow these regions, which can exacerbate neural compression. It is possible that by limiting extension, the interspinous spacer allows the injectate to more readily enter the stenotic regions and act on the irritated nerve roots.

A second explanation is that the postimplant epidural injection helped reduce inflammation associated with the implantation procedure itself. It is important to consider that the procedure may have irritated the nerves in stenotic areas, as some manipulation of the spine and surrounding tissue is expected. Repeat epidural injections to these areas may have reduced any inflammation brought about by implantation of the spacer. In both cases, repeat postimplant epidural injection has shown efficacy in further reducing symptoms of neurogenic claudication.

\section{CONCLUSION}

In conclusion, we present a series of cases in which patients with persistent neurogenic claudication have demonstrated improved responses to epidural steroid injections after interspinous spacer implantation. This is likely attributed to the spacer allowing the injectate to permeate stenotic areas and/or reducing postprocedure inflammation. Postimplant epidural steroid injections should be considered in patients with persistent symptoms. Furthermore, additional studies should be performed to further investigate possible mechanisms and responses to epidural steroid injections following interspinous spacer implantation.

\section{Acknowledgments}

Authors' Contributions: P.S. provided literature review and authoring of the manuscript. S.A. provided data collection and editorial assistance. The authors have no conflicts of interests nor any disclosures. No funding or financial compensation was received for the authoring of this case series. 


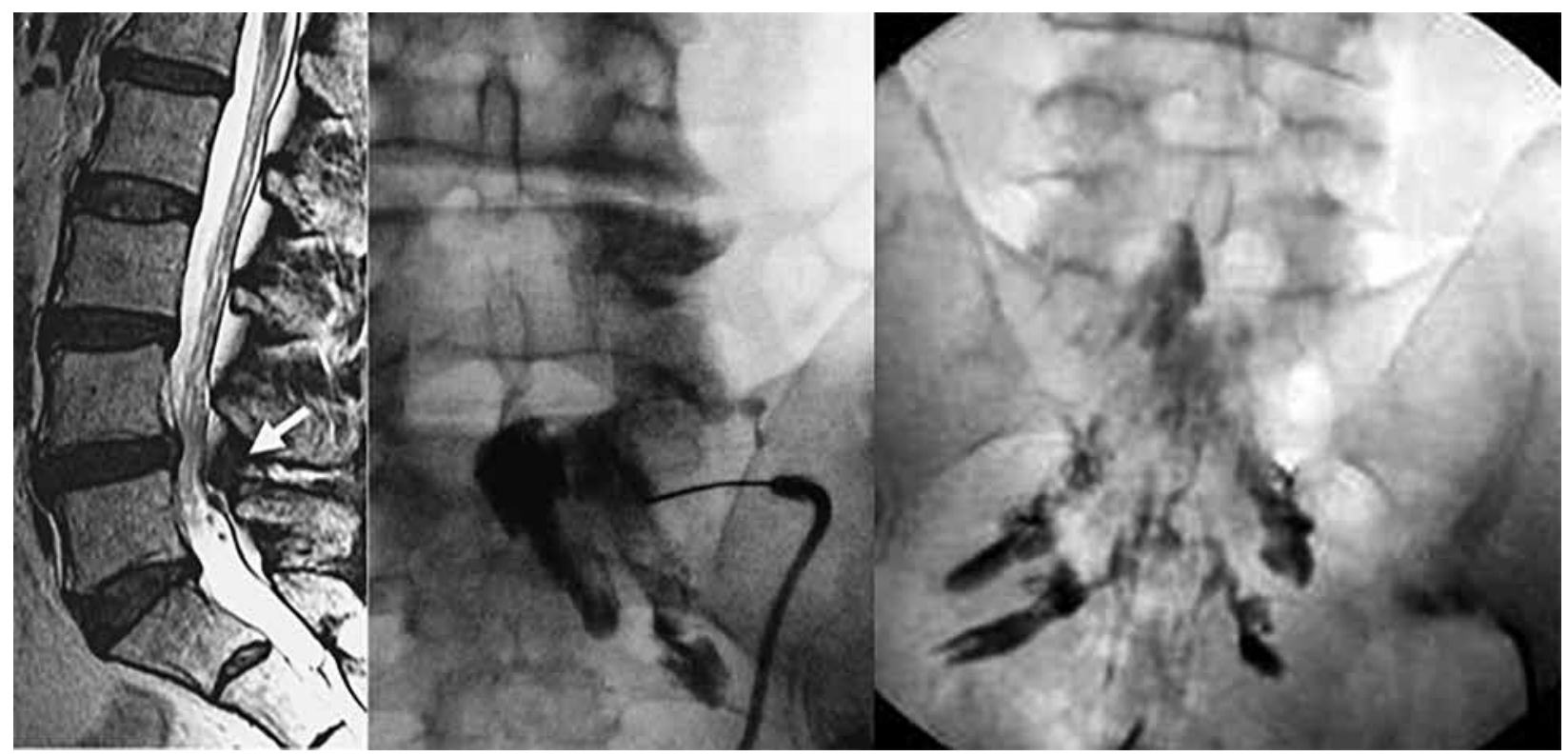

Fig. 2. MRI sagittal view of a stenotic lumbar spine (left). Preimplant fluoroscopic epidurogram AP views of the lumbar region with contrast medium (middle and right). Note the limited superior spread near the stenotic regions with interlaminar and caudal approaches.

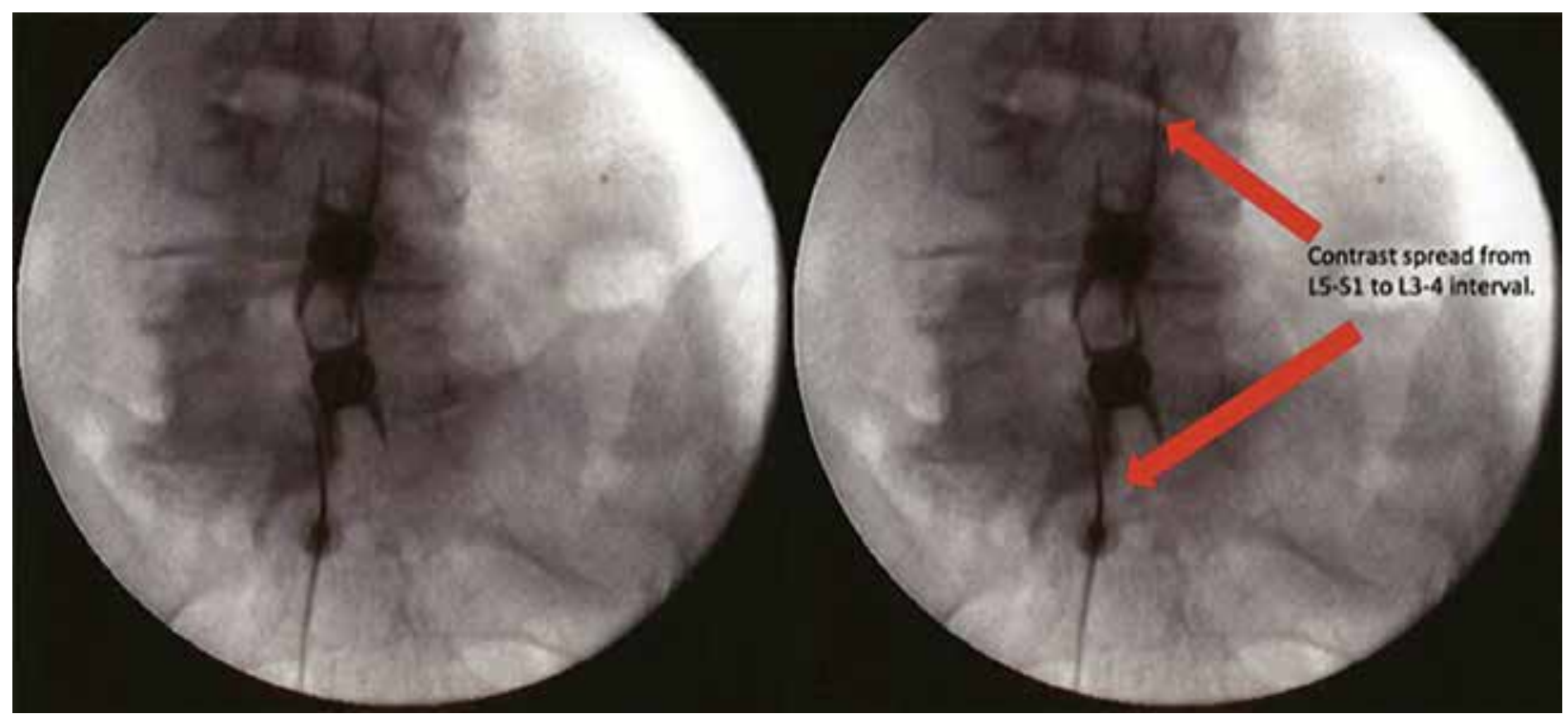

Fig. 3. Postimplant fluoroscopic epidurogram AP views of the lumbar spine with contrast medium. Note the increased superior spread of contrast medium (right; red arrows).

Abbreviations: AP, anteroposterior; MRI, magnetic resonance imaging. 


\section{REFERENCES}

1. Lurie J, Tomkins-Lane C. Management of lumbar spinal stenosis. BMJ 2016; 352:h6234.

2. Hall S, Bartleson JD, Onofrio BM, Baker Jr HL, Okazaki H, O’Duffy JD. Lumbar spinal stenosis. Clinical features, diagnostic procedures, and results of surgical treatment in 68 patients. Ann Intern Med 1985; 103:271-275.

3. Maus TP. Imaging of spinal stenosis: Neurogenic intermittent claudication and cervical spondylotic myelopathy. Radiol Clin North Am 2012; 50:651-679.

4. Amundsen $\mathrm{T}$, Weber H, Lilleas F, Nordal HJ, Abdelnoor M, Magnaes $B$. Lumbar spinal stenosis: Clinical and radiologic features. Spine (Phila Pa 1976) 1995; 20:1178-1186.

5. Issack PS, Cunningham ME, Pumberger $M$, Hughes $A P$, Cammisa Jr FP. Degenerative lumbar spinal stenosis: Evaluation and management. J Am Acad Orthop Surg 2012; 20:527-535.

6. Ciocon JO, Galindo-Ciocon D, Amaranath L, Galindo D. Caudal epidural blocks for elderly patients with lumbar canal stenosis. J Am Geriatr Soc 1994; 42:593-596.

7. Lauryssen C. Appropriate selection of patients with lumbar spinal stenosis for interspinous process decompression with the X STOP device. Neurosurg Focus 2007; 22:E5.

8. Loguidice V, Bini W, Shabat S, Miller LE, Block JE. Rationale, design and clinical performance of the Superion(R) Interspinous Spacer: A minimally invasive implant for treatment of lumbar spinal stenosis. Expert Rev Med Devices 2011; 8:419-426.

9. Zini C, Bellini M, Masala S, Marcia S. Percutaneous interspinous spacer in spinal-canal-stenosis treatment: Pros and cons. Medicina
(Kaunas) 2019; 55:381.

10. Meyer B, Baranto A, Schils F, et al; NICE Trial Study Group. Percutaneous interspinous spacer vs decompression in patients with neurogenic claudication: An alternative in selected patients? Neurosurgery 2018; 82:621-629.

11. Zucherman JF, Hsu KY, Hartjen CA, et al. A multicenter, prospective, randomized trial evaluating the $X$ STOP interspinous process decompression system for the treatment of neurogenic intermittent claudication: Two-year follow-up results. Spine (Phila Pa 1976) 2005; 30:1351-1358

12. Kondrashov DG, Hannibal M, Hsu KY, Zucherman JF. Interspinous process decompression with the X-STOP device for lumbar spinal stenosis: A 4-year follow-up study. J Spinal Disord Tech 2006; 19:323-327.

13. Nunley PD, Patel VV, Orndorff DG, Lavelle WF, Block JE, Geisler $\mathrm{FH}$. Five-year durability of stand-alone interspinous process decompression for lumbar spinal stenosis. Clin Interv Aging 2017; 12:1409-1417.

14. Patel VV, Whang PG, Haley TR, et al. Superion interspinous process spacer for intermittent neurogenic claudication secondary to moderate lumbar spinal stenosis: Two-year results from a randomized controlled FDA-IDE pivotal trial. Spine (Phila Pa 1976) 2015; 40:275-282

15. Nunley PD, Deer TR, Benyamin RM, Staats PS, Block JE. Interspinous process decompression is associated with a reduction in opioid analgesia in patients with lumbar spinal stenosis. J Pain Res 2018; 11:2943-2948. 
